\title{
ATLAS Pixel Detector Commissioning using Cosmics Rays
}

\author{
Daniel Dobos ${ }^{1}$ for the ATLAS Pixel Detector Collaboration \\ CERN - European Organization for Nuclear Research \\ Geneva, Switzerland \\ E-mail: daniel.dobosecern.ch
}

The Pixel detector of the LHC experiment ATLAS is an 80 million channels silicon tracking system designed to detect charged tracks and secondary vertices with very high precision. To verify that the integrated assembly will perform as expected subsequent to installation into the experimental area, a fraction $(\sim 8 \%)$ of the detector, the DAQ readout chain and the requisite ancillary services has been assembled and operated in a large-scale system test setting. An overview of the system and results from these tests are presented. Cosmic muon data has been taken with the setup to measure the trigger performance, check the alignment and test the reconstruction chain. All aspects of the system test, including the detector control and safety system, the monitoring system and the DAQ system, the techniques for calibrating the detector and the analysis of noise tests and cosmics data are illustrated.

The 16th International Workshop on Vertex detectors

Lake Placid, NY, USA

23-28 September, 2007

$1 \quad$ Speaker 


\section{Introduction}

The design of the ATLAS Pixel detector is driven by the requirements resulting from the LHC proton-proton collisions at a centre-of-mass energy of $14 \mathrm{TeV}$, a bunch-crossing frequency of $40 \mathrm{MHz}$ and a design luminosity of $10^{34} \mathrm{~cm}^{-2} \mathrm{~s}^{-1}$.

In order to measure the tracks of about 1000 charged particles created at each bunchcrossing with a high impact parameter and vertex resolution up to $12 \mu \mathrm{m}$, high granularity and low mass of the detector are required. To meet the low interaction length aims of $10 \% \mathrm{X}_{0}$ thinned readout electronics $(190 \mu \mathrm{m})$, carbon support structures, aluminium cables and cooling tubes for an evaporative cooling system need to be used. The placing close to the interaction point demands a high radiation dose tolerance up to $10^{15} \mathrm{n}_{\mathrm{eq}(1 \mathrm{MeV})} \mathrm{cm}^{-2}$ requiring radiation hard electronic designs and a low operating temperature. High time resolution with fast preamplifier rise times is necessary to guarantee correct bunch-crossing assignment. High occupancy together with a long trigger decision time ( $2 \mu$ s Lvl-1 trigger latency) requires on-detector zero suppression and hit data buffering.

\section{ATLAS Pixel Detector}

The Pixel detector [1] (Fig.1) is centred on the interaction point and consists of three barrel layers $(\mathrm{r}=5,9$ and $12 \mathrm{~cm})$ and two endcaps with three disks each on both faces of the barrel section. It provides three space points for the pseudorapidity region $|\eta| \leq 2.5$. Carbon support structures with integrated $\mathrm{C}_{3} \mathrm{~F}_{8}$ evaporative cooling

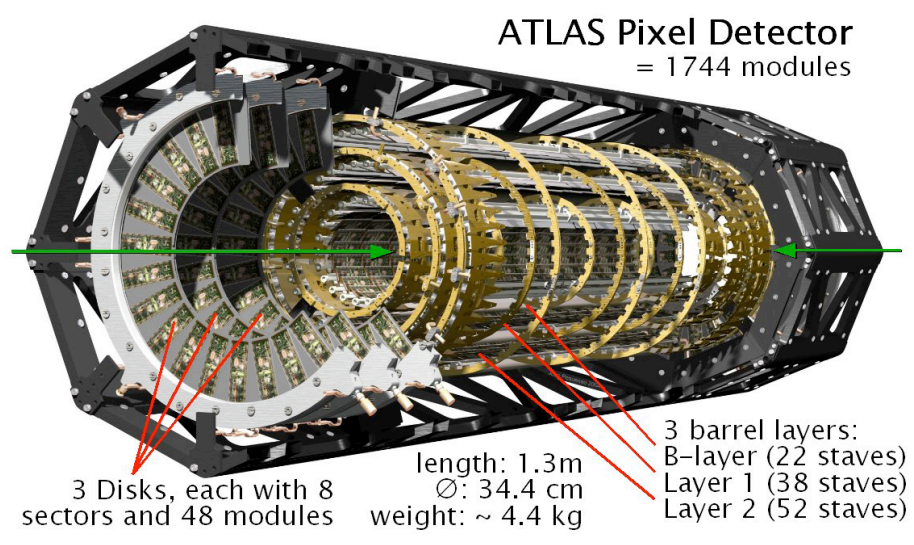

Fig. 1. Schematic view of the ATLAS Pixel detector with three barrel layers and two endcaps with three disks each. tubes support 1744 identical Pixel modules, which together form an active silicon area of about $1.8 \mathrm{~m}^{2}$ and provide 80 million readout channels. The cooling system keeps the modules with an overall power load of $10 \mathrm{~kW}$ at an operating temperature of about $-10^{\circ} \mathrm{C}$ to prevent reverse annealing effects after radiation damage of the silicon sensors.

\subsection{Pixel Module}

Pixel modules (Fig. 2) are assemblies of a $250 \mu \mathrm{m}$ thick oxygenated silicon sensor [2], 16 front-end readout ASICs [3], a Module Control Chip (MCC) and a flexible kapton PCB (flex) for routing between the components. The sensor is structured in 47.232 pixel cells. Each sensor pixel implantation of $50 \times 400 \mu \mathrm{m}$ size is connected to a preamplifier readout channel on one of the front-end chips via fine-pitch $(50 \mu \mathrm{m})$ solder or indium flip-chip bump bonds. The front-end pixels each contain individually adjustable charge sensitive preamplifier, threshold and digitization circuits. Hit data are zero suppressed and buffered in the front-end chips until a Lvl1 trigger for the particular event arrives or an adjustable latency passed. Front-end chips have 
wire bond interconnections to the multilayer flex glued to the sensor. Besides signal routing lines between the frontends and the MCC, the flex contains SMD capacitors for voltage decoupling and a NTC resistor for temperature measurement. The MCC distributes offdetector electronics Timing, Trigger and Control (TTC) signals to the frontends and performs a module based hit data event building. A cross-sectional view of the Pixel module is shown as well in Figure 2.

The schematic layout of a sensor pixel and the connected front-end pixel is illustrated in Figure 3. FE channels incorporate a fast high gain preamplifier with a feedback capacitor. A DC feedback scheme can compensate

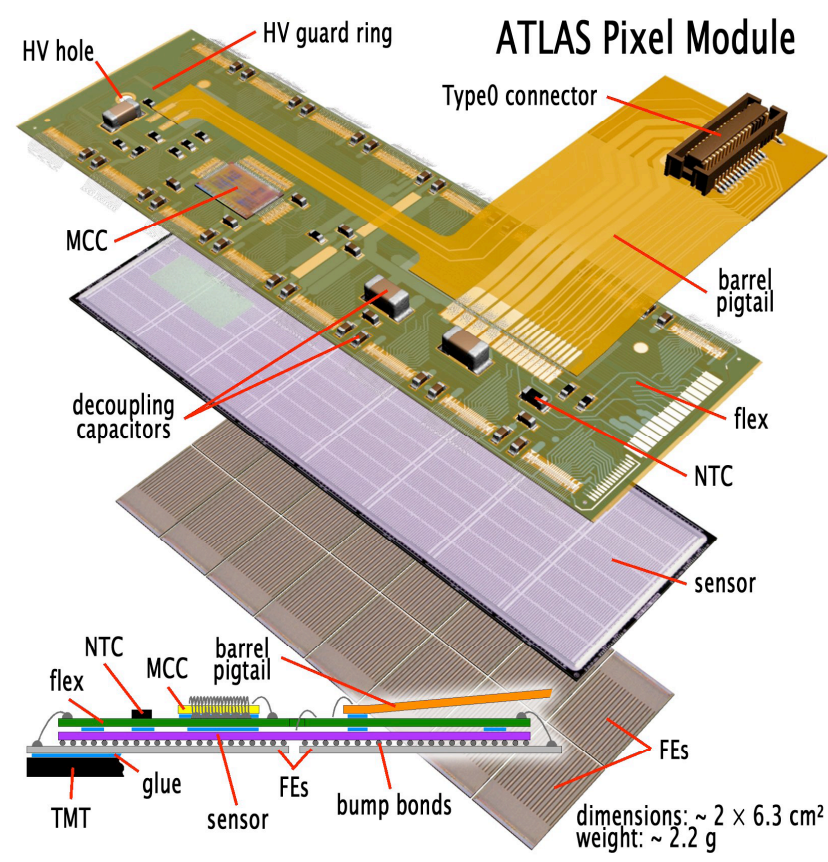

Fig. 2. Schematic and cross-sectional view of an ATLAS Pixel module sensor leakage currents of up to $100 \mathrm{nA}$ on the preamplifier input. The feedback capacitor is discharged by an adjustable constant current source, so that a nearly triangular pulse shape is obtained. A DC-coupled second stage and a fast differential discriminator (adjustable in $75 \mathrm{e}^{-}$ steps) are following the preamplifier for zero suppression. Due to the constant discharge of the feedback capacitor the discriminator output signal Time-Over-Threshold (TOT) is proportional to the charge deposited in the sensor pixel. The feedback is adjusted so that it is a good

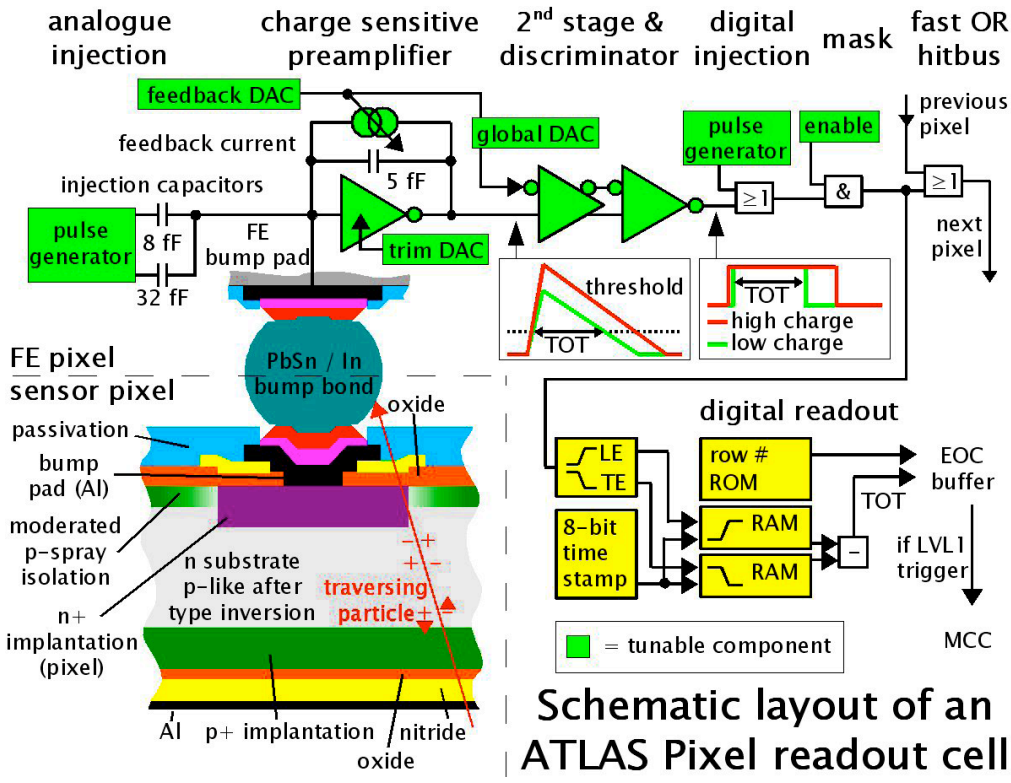

Fig. 3. Cross-sectional view of a sensor pixel and block diagram of the front-end readout pixel cell compromise between high charge resolution and a small pixel dead time. The length of the TOT pulse is measured in units of the 40 $\mathrm{MHz}$ bunch crossing clock with an 8-bit Gray-coded counter. The TOT is calculated from the time stamps of the leading and trailing signal edges and stored together with a pixel identifier in the End-OfColumn (EOC) region of the front-ends. They are transferred to the MCC only if a Lvl-1 trigger arrives 
within an adjustable delay. Analogue and digital injection circuits are implemented for test and calibration purposes.

\subsection{Optical Data Transmission}

The data and supply lines of a module are routed combined in a lowmass aluminium (barrel) or copper (endcap) micro cable about $1 \mathrm{~m}$ long to a so-called Patch Panel 0 (PP0) mounted on the service quarter panels on both sides of the detector. In addition to supply lines the PP0s contain optoboards [4] (Fig. 4) for conversions between optical and differential electrical signals.

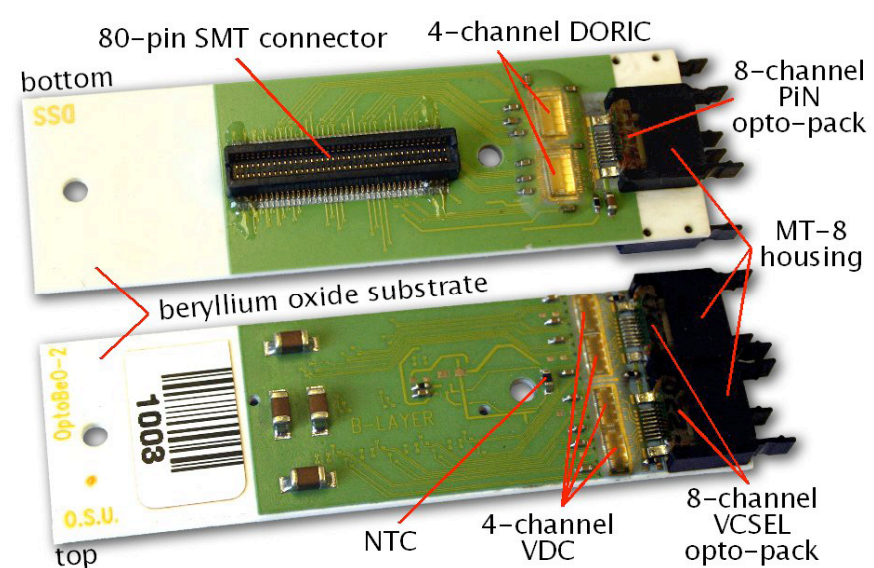

Fig. 4. Photographic views of both sides of an optoboard

Unidirectional optical links are used for all data transfer from and to the modules to avoid problems caused by ground loops and electromagnetic interferences. Additionally they have the advantage of high bandwidth and low radiation length.

An optoboard receives BiPhase Mark (BPM) encoded TTC signals for 6 or 7 modules on individual fibres using epitaxial silicon PiN diodes. Digital Opto-Receiver Integrated Circuit (DORIC) chips are used to recover the bunch-crossing clock as well as the $40 \mathrm{Mbit} / \mathrm{s}$ trigger and control data stream. Their gain stage incorporates internal feedback to adjust the threshold and a delay-lock-loop to maintain 50\% duty cycle over the entire current input range. The differential data output lines of the modules are converted by a Vertical Cavity Surface Emitting Laser (VCSEL) Driver Chip (VDC) into single-ended current signals driving fast rise and fall time high light output GaAs $850 \mathrm{~nm}$ VCSELs. The amplitudes of the VCSEL currents can be set together for all 6 or 7 channels by a single control current determined by an external control voltage $\left(\mathrm{V}_{\text {Iset }}\right)$. One or two (for dual-link mode of the innermost barrel layer) fibres per module are used for data transmission to the off-detector electronics (see Fig. 5).

At the off-detector side [5] the approximately $80 \mathrm{~m}$ long fibres are connected to optical

\section{Optical Link Parameters}

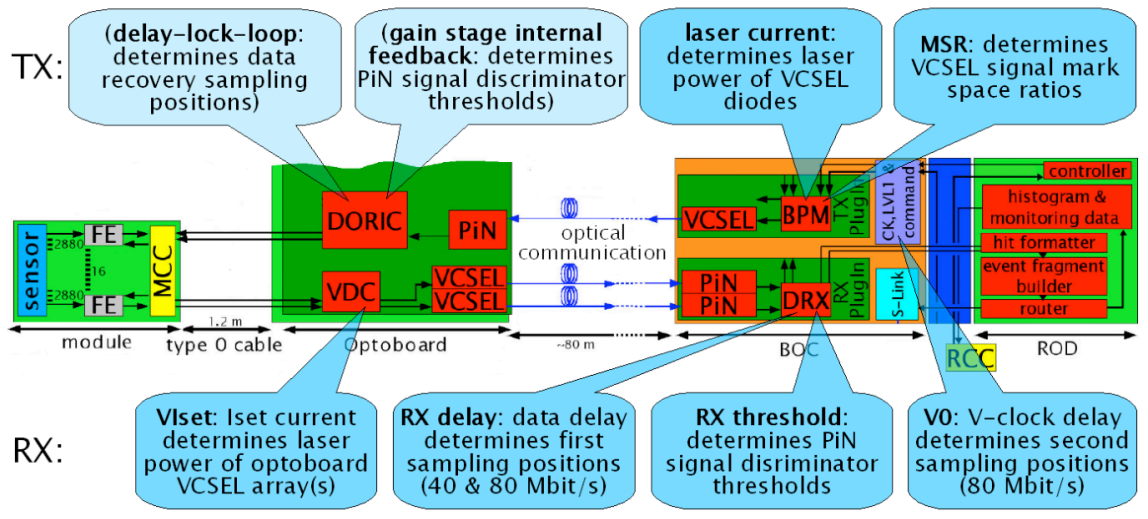

Fig. 5. Pixel detector readout chain and involved optical link parameters interface Back Of Crate (BOC) cards. Because the clock is not encoded in the data stream the phase between the BOC clock and the arriving data (RX delay) require tuning. So does the PiN threshold (RX threshold). For 
$80 \mathrm{Mbit} / \mathrm{s}$ data transmission mode the second data sampling position is determined by another (V0) clock delay. On the transmission side the laser currents and Mark Space Ratios of optical links can be adjusted individually. Each BOC is paired with a ReadOut Driver (ROD), which is the main Pixel module control and data receiver electronics, sends calibration commands to the modules as well as performs event fragment building and online monitoring.

\section{System Test}

An $\sim 8 \%$ fraction of the detector system was assembled and operated in a laboratory setup in the ATLAS Inner detector assembly clean room at CERN from September 2006 to January 2007. The aim of the System Test [6],[7] was to verify that the Pixel detector integrated assembly, its DAQ readout chain and its final ancillary services perform and interact as expected. Even though all on- and off-detector components have been tested on their production sites and in smaller setups in various labs the interaction of all detector elements and the scalability of the service and readout chain was reasonably tested for the first time in the System Test. The scalability of the system was checked and stress tests were performed. It was used to test and debug the hardware and software components in parallel and allowed operator feedback to the experts. The detector was operated under realistic conditions in long-run mode to test its stability and to allow tracking of its performance over a long term. An additional important goal was to define and qualify procedures for the operation, calibration and data taking in the experimental setup. Scintillators for triggering of cosmic were integrated in order to investigate and commission the data taking operation, the software as well as the trigger components.

\subsection{System Test Setup}

The setup (Fig. 6) allowed to operate one of the Pixel endcaps with 144 modules through a prototype service quarter panel (SQP) with 24 attached optoboards under realistic environmental conditions. It is the biggest operated ATLAS Pixel system so far. The endcap and the optoboards on the SQP were chilled with the evaporative cooling system. Production components could be used for almost all service elements.

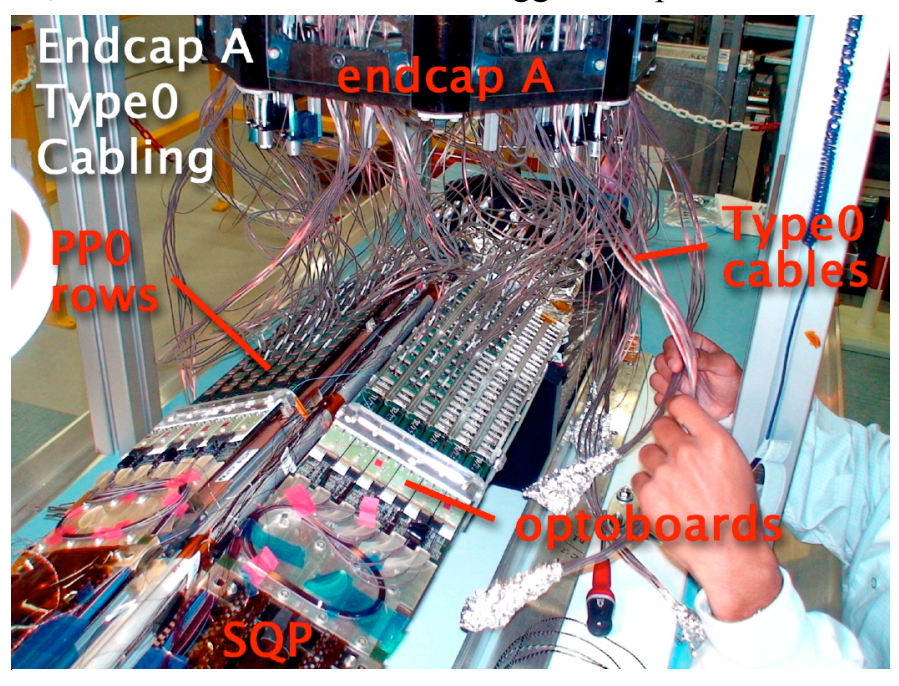

Fig. 6. System Test setup: Endcap A connected by Type0 cables to PP0s with optoboards on the SQP

A schematic view of the readout, supply, control, monitoring and interlock systems is shown in Figure 7. A description of the readout chain with electrical differential data transmission between modules and optoboards as well as optical data transmission between optoboards and BOCS is given in Section 2.

For high current module and optoboard supply lines VDD, VDDA and VVDC the distance of about $100 \mathrm{~m}$ to the power supplies in the counting room would lead to intolerably high 
voltage drops and thus high power dissipation in the service cables. A compensation at the power supplies would be risky, since a sudden current decrease could lead to voltages above the $0.25 \mu \mathrm{m}$ DSM CMOS breakdown voltage of $4 \mathrm{~V}$. Therefore power supply regulator stations are used closer to the detector (PP2). The voltage drop over the



Fig. 7. Readout, supply, control, monitoring and interlock systems of the System Test setup remaining $15 \mathrm{~m}$ supply cable is still significant and is compensated by remote sensing. The voltage drops of the low current optoboard supplies $\mathrm{V}_{\text {Iset }}, \mathrm{V}_{\mathrm{PiN}}$ and of the sensor bias voltage are negligible, no remote sensing is necessary and power supplies in the counting room can be used directly.

On-detector electronics temperatures are monitored with NTC resistors, readout by offdetector Building Block Interlock and Monitoring (BBIM) units, which incorporate temperature interlock thresholds. A dedicated pure hardware negative-logic interlock system is aimed to switch off only devices directly involved in the interlock situation. Environmental humidity and temperature sensors are readout by Building Block Monitoring (BBM) units. The slow-control and monitoring of the power supply and interlock components as well as the monitoring of the

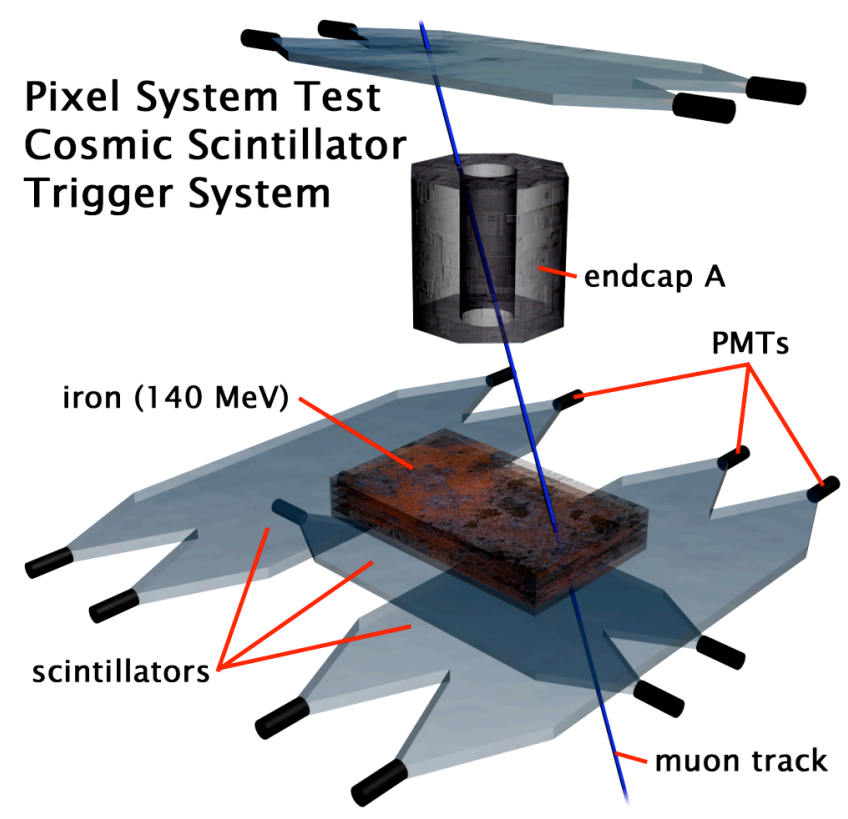

Fig. 8. Schematic view of the System Test scintillator trigger system for cosmic data taking with the endcap A detector voltages, currents and environmental sensors are tasks of the Detector Control System (DCS).

For triggering on cosmic muons four scintillator panels with four Photo Multiplier Tubes (PMT) each were arranged around the endcap (Fig. 8). One was placed above and three beneath the endcap. To avoid triggering on low momentum $(<140 \mathrm{MeV})$ muons a block of iron was placed above the lower scintillators. On coincidence of the upper and one of the lower scintillator panels a trigger was distributed through a Local Trigger Processor (LTP) crate to the TTC Interface Modules (TIM) of two ROD 
crates. Module hit data was sent from the RODs via S-Link transmission to a ReadOut Subsystem (ROS) and combined to event fragments for storage.

\section{System Test Results}

An automated service test system was developed to verify the correct function of the entire service chain including interlocks and the corresponding connectivity information in the slow control and detector calibration software. It was qualified with the System Test setup for the service tests in the experiment before the connection of the detector. These tests are ongoing at the time of writing and are expected to be finished by the end of 2007. Experience with the biphase evaporative cooling system was gained and procedures for its operation were defined [9]. More details of the cooling performance results can be found in [7] and [8]. All service and cooling component fulfil the requirement of the detector.

\subsection{Optical Communication Tuning}

An error-free data transmission via the optical link between the BOC and the optoboards (see section 2.2) is essential to guarantee precise module configuration and triggering as well as to allow a sensible readout of module hit and calibration information. Due to the delay-lockloop and threshold adjustment circuits in the optoboard the TTC data transmission showed reliable performance with standard BOC laser currents and MSR settings and no further tuning was necessary.

For the module data transmission the laser output power of the VCSEL array is controlled by one external reference voltage $\left(\mathrm{V}_{\text {Iset }}\right)$ for all 8 optical channels of the optoboard. It needs to be adjusted in a way, that the phase between the BOC $40 \mathrm{MHz}$ sampling and the incoming data ( $\mathrm{RX}$ delay) as well as the threshold applied to the BOC Pin output signal (RX threshold) can be adjusted to the most stable working points. An example of a so-called BOC scan, used for RX threshold and RX delay tuning, is shown in Figure 9.

The amount of transmission errors of a welldefined bit-pattern is colour-coded as a function of the RX threshold and RX delay. The shape of the error free region corresponds to the rising edge (blue) and falling edge (green) of the laser signal shape (signal edges swapped in plot due to increasing delay). It shows horizontal delayindependent error-bands at low and high threshold, which correspond to sampling the signal with a threshold below the baseline and with a threshold above the high value. The vertical error-band corresponds to sampling in the rising and falling edge of the signal. With increasing $\mathrm{V}_{\text {Iset }}$ the signal amplitude and the baseline increase linearly. The slope of the lower threshold error-band is only about a fourth of the upper error-band slope. Since

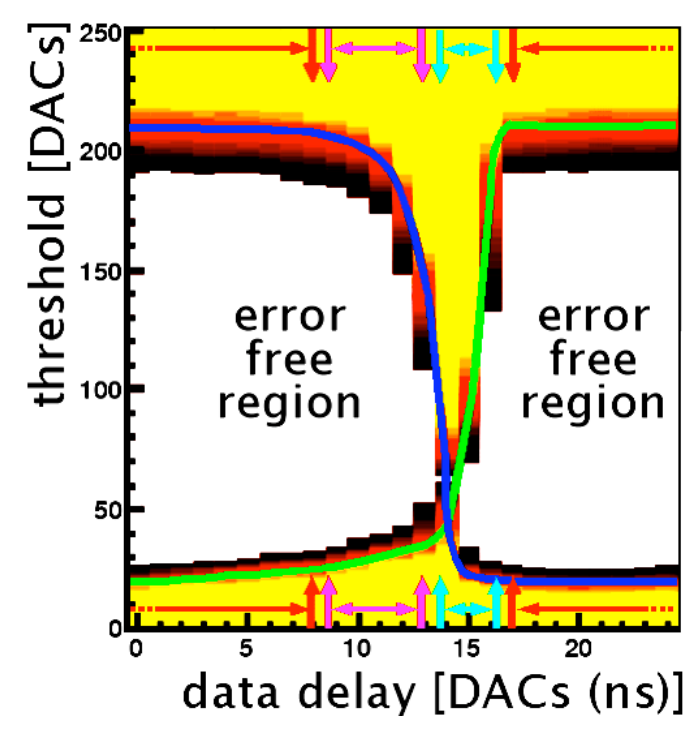

Fig. 9. BOC scan example for one optoboard channel (colour-coded bit-errors) 
the lower error-band is in the dynamic range of the RX threshold even for high laser output powers it can be used as a measure for the laser power.

With systematic studies of the laser signal shape, output power and BOC scans under various operating conditions, few problematic features of the optoboard were discovered. For few optoboards a significant channel-to-channel light output power spread of the same VCSEL array was observed. Due to the single power-regulating voltage $\mathrm{V}_{\text {Iset }}$ for an array in extreme cases this led to light output powers outside the dynamic range of the BOC receiver for few channels. Quality assurance procedures were improved to exclude the usage of high laser power spread optoboards for the final detector assembly. With optoboard temperatures below their design operating temperature of $10^{\circ} \mathrm{C}$ the power spread increases and the output power decreases, also leading to the mentioned tuning problems. A sophisticated optoboard temperature control system was implemented in the final detector design and additional quality assurance measurements below design temperature were included. A low subset of channels showed a slow rise of the laser power after a longer time of inactivity, referred to as slow turnon. A typical example of a slow turn-on channel measured with the BOC scan bit-pattern and an optical probe are shown in Figure 10. Feedback from the system test led to a dedicated slow turn-on quality assurance measurement, which successfully excluded the integration of optoboards with slow turn-on channels in the final detector.

According to the observed problems an optical link parameter tuning procedure to determine the most stable optical link working point was developed and qualified under realistic conditions with the System Test setup.


Fig. 10. Slow turn-on effect of an optoboard channel in the BOC scan measurement (left) and directly measured with an optical probe (right)

\subsection{Module Tuning Performance}

The threshold and noise of a pixel are measured with repeated charge injections into the pixel preamplifier by a front-end chip chopper circuit (see Fig. 3). A threshold scan measures the discriminator response (fraction of hits to injections) versus the known injected charge. For an ideal discriminator a step function at the adjusted threshold is expected (see Fig. 11). With 
noise in the readout circuits taken into account the discriminator output is described by a convolution of the stepfunction with the Gaussian pixel noise, i.e. the Gaussian error-function. With an Scurve fit the threshold is determined as the $50 \%$ efficiency value as well as the one sigma noise from the $30 \%$ and $70 \%$ efficiency values (see Fig. 11). In the $250 \mu \mathrm{m}$ silicon sensor a MIP has a most probable energy loss of $19 \mathrm{ke}^{-}$, about $10 \mathrm{ke}^{-}$ with charge sharing and goes down to about 5 ke- after life-time dose irradiation. The

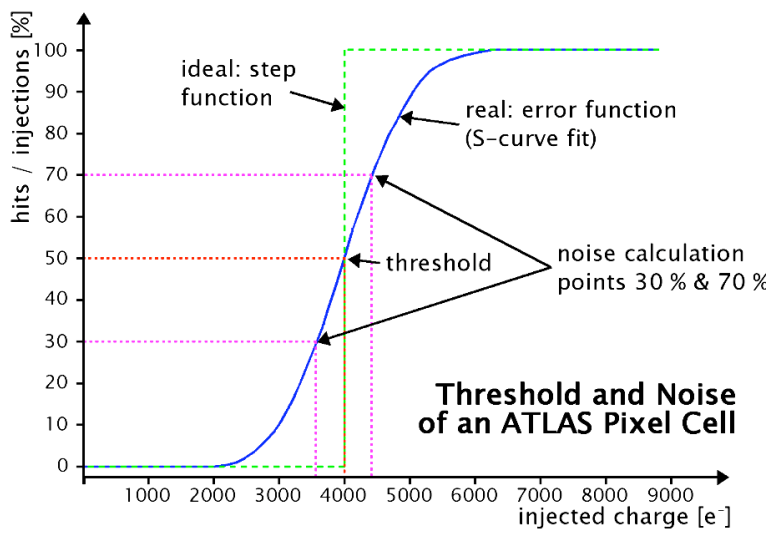

Fig. 11. Ideal step-function and real Gaussian error-function result of a threshold scan
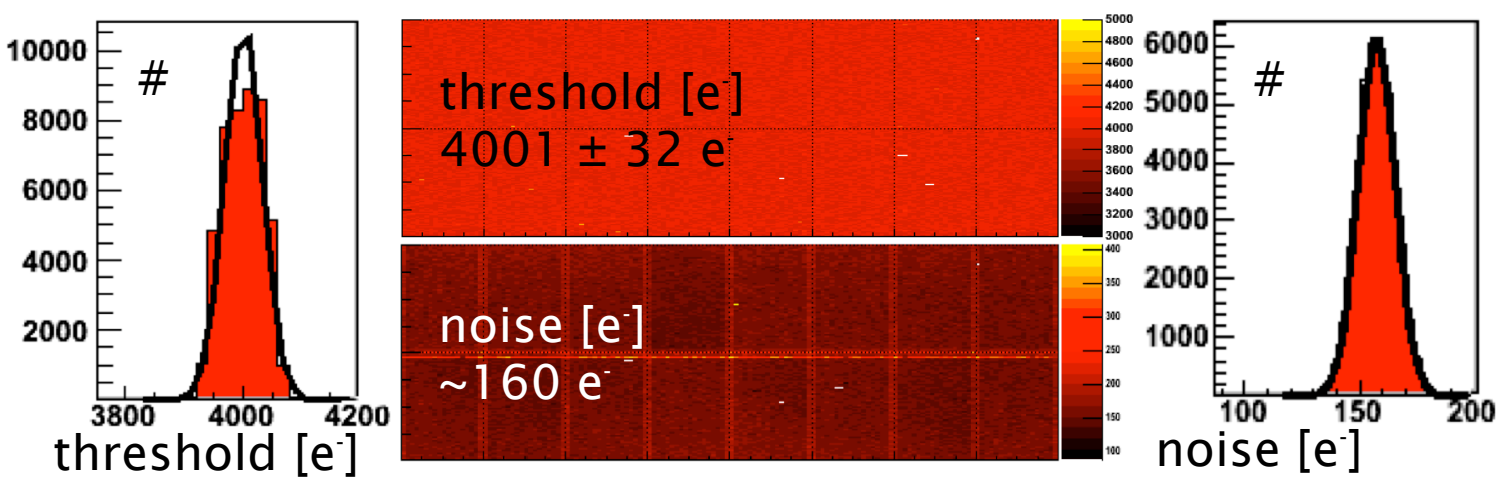

Fig. 12. Distribution and maps of pixel thresholds and noise for one module


Fig. 13. Module threshold production and System Test distributions (left) and module noise System Test versus production correlation (right) 
pixel discriminator thresholds are adjusted to ensure a homogeneous detection probability at a target threshold of $4 \mathrm{ke}^{-}$. The colour-coded pixel threshold and noise maps of a tuned module as well as their distributions are shown in Figure 12. The histograms are fitted with a Gaussian distribution to determine the threshold, threshold dispersion, noise and noise dispersion of a module. Higher noise values at the front-end chip edges are caused by pixels with larger dimensions and by two pixels connected to a single preamplifier channel. Figure 13 shows the module threshold distributions of the tuning during production and in the System Test. The tuning algorithm of the final readout and calibration system shows a threshold of $4002 \pm 15 \mathrm{e}^{-}$ and a threshold dispersion of $35 \mathrm{e}^{-}$. This is significantly better than the production tuning algorithm. In the module noise correlation plot the System Test noise is systematically about $10 \mathrm{e}^{-}$higher than the production noise. For the production threshold scans only the scanned pixel preamplifiers where activated and the neighbouring deactivated whereas in the System Test all amplifiers were active. The latter is known to have systematically higher noise due to crosstalk between the pixel circuits. The System Test results verify an excellent threshold tunability of the Pixel modules with the final readout system and show no indication for a significant noise increase with the final detector services.

\subsection{Noise Occupancy and Sensor Depletion Voltage}

Annealing and reverse annealing effects after irradiation have a significant impact on the depletion voltage of the silicon sensor. Together with the charge collection efficiency they dominate the lifetime of the detector. A permanent monitoring of the depletion voltage is therefore necessary especially in the first commissioning and irradiation phase. It allows to verify and constantly update the detector lifetime predictions.

The depletion voltage changes with increasing irradiation of the sensor. Therefore, it is a measure for the integrated radiation dose. Before the sensor bulk type inversion only full depletion allows a sensible space resolved particle detection, because below full depletion the pixels are electrically shorted. With increasing radiation dose the depletion voltage decreases until type inversion occurs. Afterwards the depletion voltage increases with radiation dose and requires an increase of the bias voltage up to the maximal nominal operation voltage of $600 \mathrm{~V}$. Beyond this point the depletion depth decreases, which can result in a decrease of the charge collection efficiency.

During data taking phases the charge collection efficiency can be used to measure the depletion depth. Via the saturation of the depletion depth (or charge collection efficiency) with increasing sensor bias voltage the depletion voltage can be measured. Before type inversion the depletion zone grows towards the pixel side. If the sensor bias voltage is below the depletion voltage the pixels are shorted, the preamplifiers see a high capacitive load resulting in high pixel noise and noise occupancy values. Therefore a measurement of the module noise occupancy versus sensor bias voltage can be used to measure the depletion voltage and has the advantage (over the charge collection efficiency method) that it can be performed in maintenance periods without beam collisions. The System Test setup was used to study this method with a fixed trigger frequency generated by an external pulser. Noise occupancy versus sensor bias voltage curves for 117 endcap A modules are shown in Figure 14. 

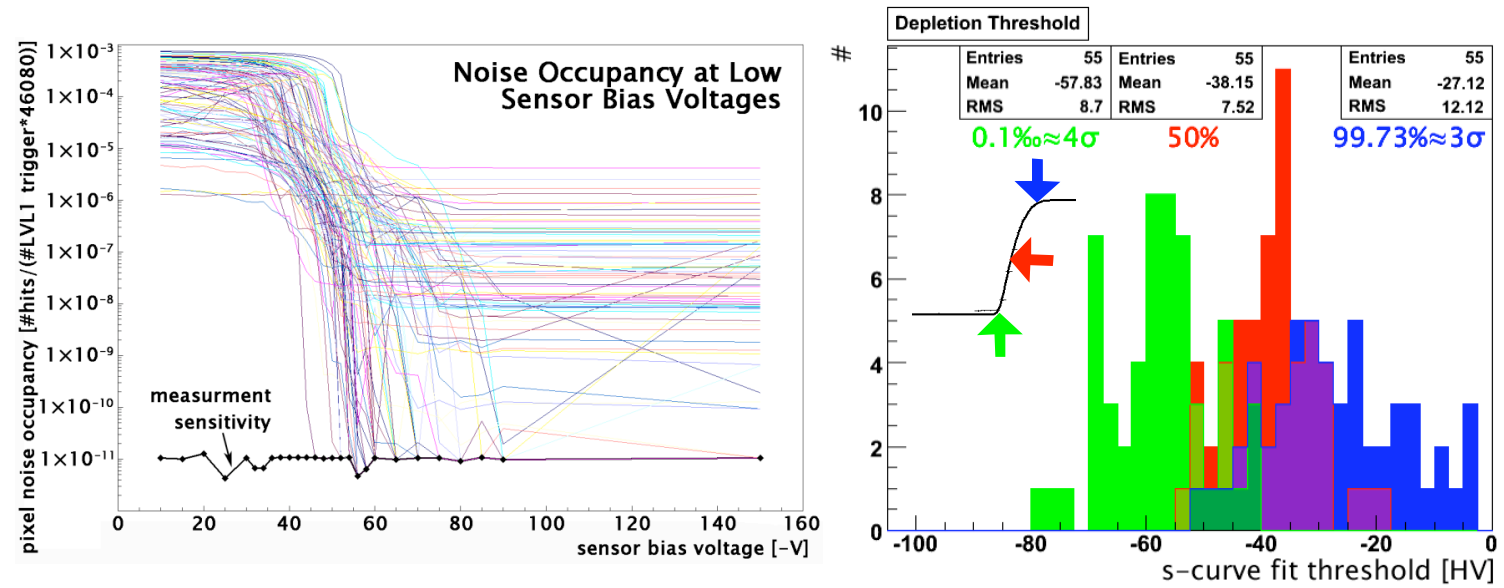

Fig. 14. Noise occupancy vs. bias voltage curves for 117 modules of endcap A (left) and 50\% (red), $4 \sigma$ (green) and $3 \sigma$ (blue) bias voltage values obtained from an error-function fit to the curves (right)

A pixel noise occupancy measurement sensitivity of $10^{-11}$ was reached with the collected statistics. In order to determine the depletion voltage the transition between the low noise occupancy at full depletion and the high noise occupancy at partial depletion is fitted with the Gaussian error-function. The depletion voltage is determined from the error-function as the $4 \sigma$ value of the mean transition voltage. This method allows a fast determination of the depletion voltage before type inversion and thus the integrated radiation dose without time-loss for data taking. It can be used to optimize the detector lifetime by steering the temperature profile of the detector during maintenance periods to take advantage of sensor annealing without the drawbacks of reverse annealing.

After sensor type inversion the measurement of the depletion voltage with the noise occupancy method is not possible. The charge collection efficiency and depletion depth saturation methods can be used further on, but only with the drawback that measurements are only possible at the expense of data taking time.

\subsection{Cosmics and Alignment}

For each scintillator trigger, i.e. the coincidence between the upper scintillator panel and any of the bottom panels, 16 consecutive Level-1 accept triggers were sent to the Pixel modules. A cosmics trigger rate of $15.7 \mathrm{~Hz}$ was measured, which agrees very well with the Monte Carlo Geant4 simulation setup expectation of $16 \mathrm{~Hz}$. The hit timing distribution of the first System Test cosmics run is presented in Figure 15. It gave the first evidence that cosmic muon hits were seen in the endcap modules with a fixed effective delay of $125 \mathrm{~ns}$ with respect to the first of the 16 Level-1 accept

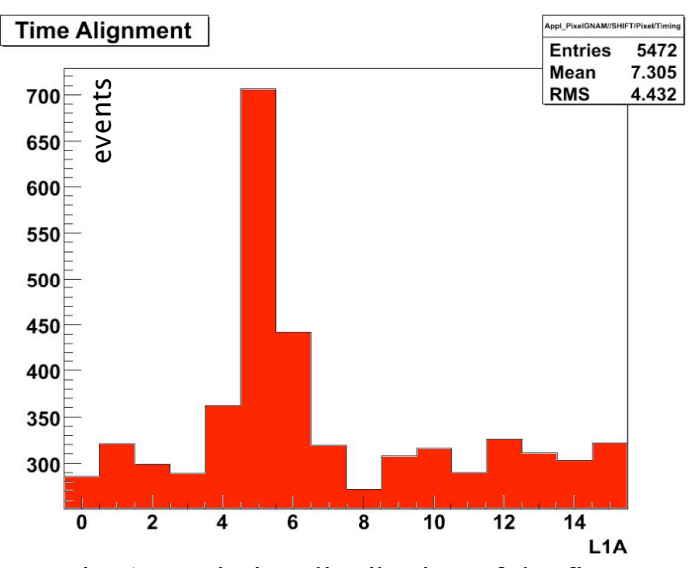

Fig. 15. Timing distribution of the first ATLAS Pixel cosmics run triggers. Off-peak entries are caused by unmasked noisy pixels since they have hits, which are 

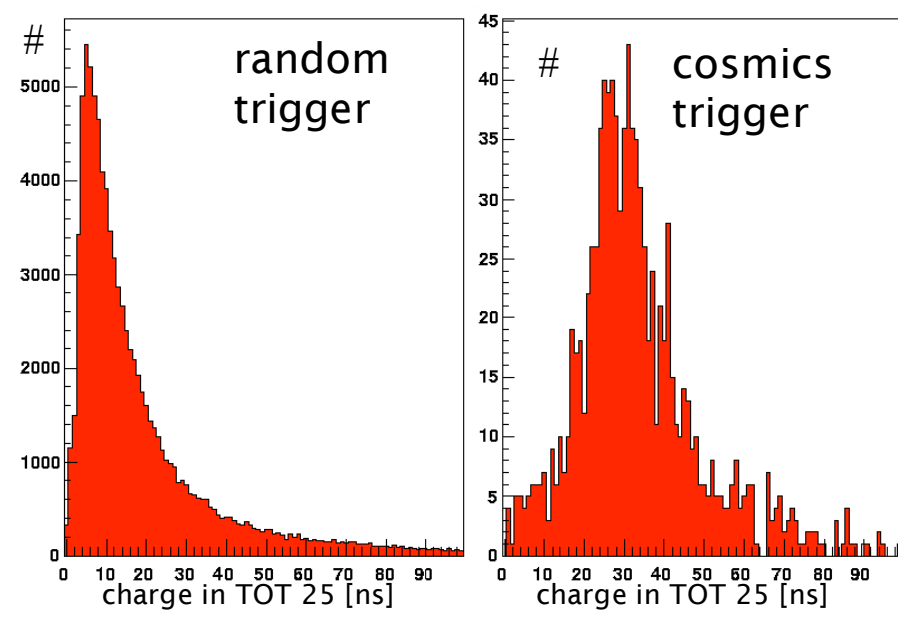

Fig. 16. Charge distributions in units of TOT for random (left) and cosmics triggers (right)

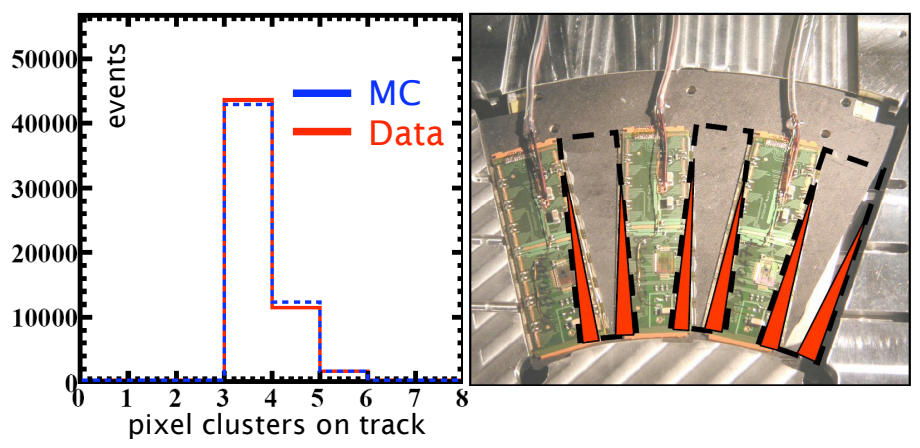

Fig. 17. Pixel clusters on track distribution comparison (left) and module overlap regions on a disk (right)

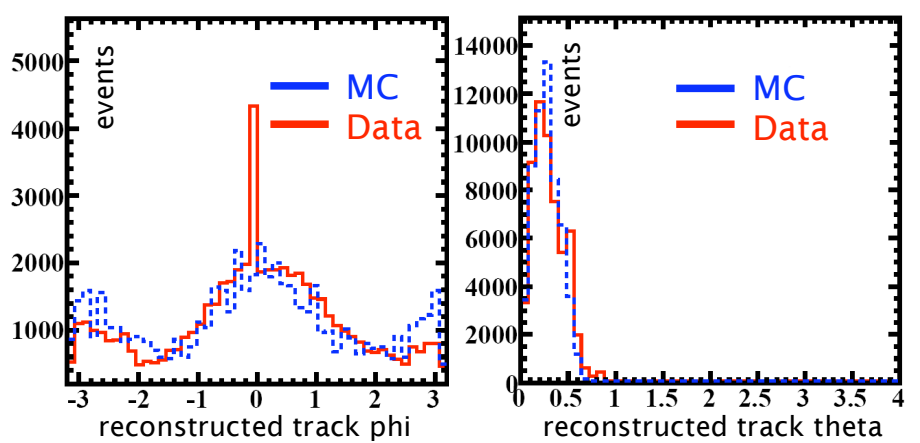

Fig. 18. Monte Carlo and data comparison of the track incident angles $\phi$ (left) and $\theta$ (right)


not correlated with the scintillator triggers. The TOT distribution of a random trigger and the cosmic trigger run are compared in Figure 16. For the cosmics trigger only hits with Level-1 accept between 4 and 6 are taken into account. With the random trigger the distribution shows the characteristic shape for noise hits with a distinct peak at TOT $=5$ and a distinct tail towards high TOT values, whereas with the cosmics trigger the TOT distributions shows the characteristic distribution of a MIP with a peak at around 30 TOT units.

In total about $10^{6}$ cosmic events were recorded and used to study the performance of clustering, tracking and alignment algorithms in the offline reconstruction software. Comparisons with the Monte Carlo simulations allowed crosschecking the offline software detector description. Figure 17 shows the clusters on track distribution for the simulation (blue) and the data (red). Three clusters are observed for tracks passing through all three endcap disks. Tracks with more than three clusters occur if a track passes

Fig. 19. Monte Carlo and data comparison of the cluster width on track (left) and the charge on track in units of time-over-threshold (right) 
through module overlap regions of a disks (see Fig. 17), contributing two clusters per disk. With tracks in the overlap regions the clustering efficiency was determined to be $99.5 \%$. The incident angles $\phi$ and $\theta$ of the track with respect to the beam axis show good agreement with Monte Carlo simulations. The phi distribution is inhomogeneous due to the asymmetric arrangement of the scintillators around the endcap. A peak in the distribution is caused by three noisy pixels, which are on a line and are repeatedly reconstructed as a track. Also the cluster width on the reconstructed track distribution shows good agreement with the Monte Carlo Simulation. Only the TOT charge distribution shows significant discrepancies (see Fig. 19). The TOT shape is simulated correctly, but a shift is caused by a wrong conversion of a TOT fit parameter measured during module production.

The tracks passing through the module overlap regions were also used to study the relative alignment between adjacent modules on different sides of a disk with a distance of $4.2 \mathrm{~mm}$ in $\mathrm{z}$ direction. Relative alignment constants obtained from the cosmics alignment were correlated with constants obtained from a survey during the assembly of the disks (see Fig. 20). With the nominal survey geometry a spatial resolution of $23 \mu \mathrm{m}$ is obtained for the short pixel direction. With a preliminary cosmics alignment the spatial resolution could be improved to $16 \mu \mathrm{m}$, which is close to the $14 \mu \mathrm{m}$ resolution expectation from the Monte Carlo simulation. For the long pixel direction the spatial resolution agrees very well with Monte Carlo prediction.

A detailed discussion of the offline cosmics and alignment studies can be found in [10].
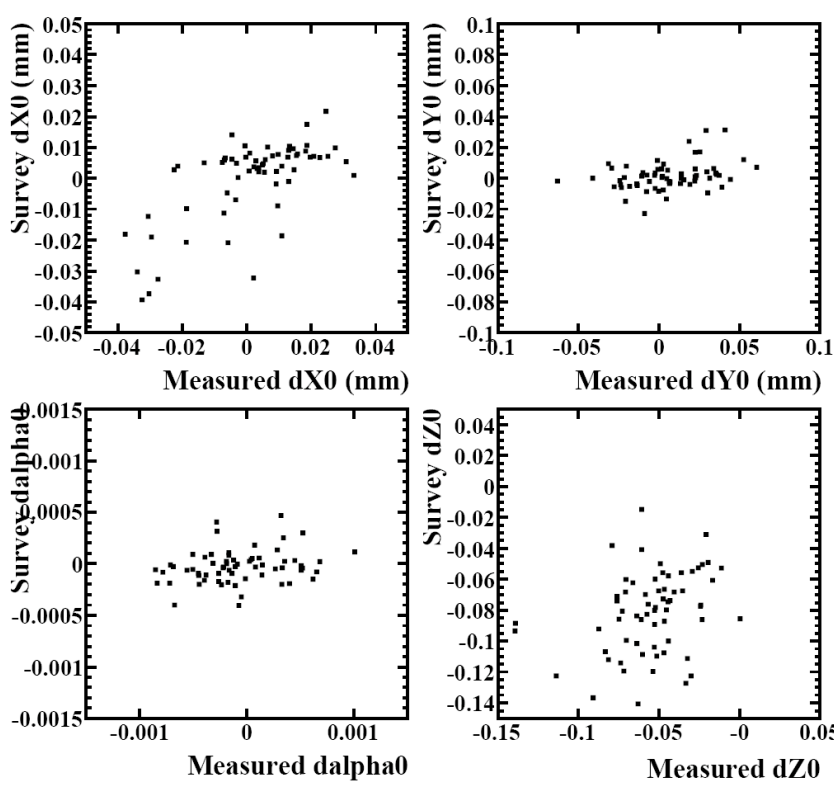

Fig. 20 Survey versus cosmics alignment for modules with more than 50 hits in the overlap region
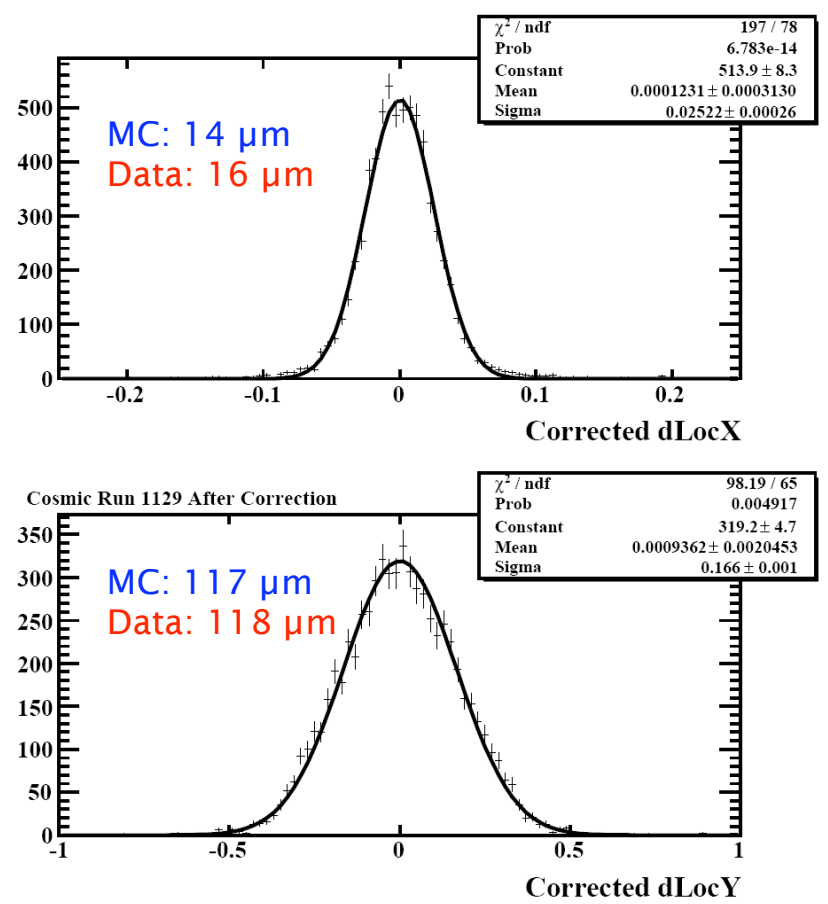

Fig. 21. Spatial resolution in the short pixel direction (top) and in the long pixel direction (bottom) with the cosmics alignment 


\section{Conclusions}

The $\sim 8 \%$ ATLAS Pixel detector System Test was a success and valuable experience for a successful commissioning and operation of the detector was gained. Various parts of the detector including cooling, services and interlock system have been validated. Driven by the System test huge development steps in online and offline software were achieved. Difficulties in the optical communication system were identified in time to take necessary quality assurance and design change actions before assembly of the final detector. Excellent detector performance (threshold, noise, noise occupancy) known from the production could be verified and no system specific problems have been observed. Monte Carlo expectations for cosmic data were confirmed and the recorded data allowed to test the entire reconstruction chain as well as to exercise alignment and resolution studies.

\section{Acknowledgments}

The presented work is the joint effort of many members of the ATLAS Pixel Detector Collaboration. I want to thank all of them.

\section{References}

[1] ATLAS Collaboration, Pixel Detector Technical Design Report, CERN-LHCC-98-143, 1998.

[2] F. Hügging, Der ATLAS Pixelsensor, PhD thesis, Dortmund University, CERN-THESIS-2001-027, 2001.

[3] K. Einsweiler, On-detector Electronics Architecture, Draft V3.0, 2003.

[4] P. Gerlach, ATLAS Pixel Optolink FDR, ATLAS project document ATL-IP-ER-007 Rev 1.0, CERN, 2003.

[5] M. Chu et al, The off-detector opto-electronics for the optical links of the ATLAS semiconductor tracker and pixel detector, Nuclear Instruments and Methods in Physics Research A 530, p. 293, 2004.

[6] D. Dobos, Commissioning Perspectives for the ATLAS Pixel Detector, $\mathrm{PhD}$ thesis, University of Dortmund, CERN-THESIS-2008-022, 2007.

[7] J. Weingarten, System Test and Noise Performance Studies at The ATLAS Pixel Detector, $\mathrm{PhD}$ thesis, University of Bonn, CERN-THESIS-2008-033, 2007.

[8] J. Weingarten, ATLAS Pixel Detector System Test and Cosmics Run, IEEE 2007 Nuclear Science Symposium and Medical Imaging Conference, Honolulu, Hawaii, submitted to Transactions of Nuclear Science.

[9] The ATLAS Pixel Group, The System Test of the ATLAS Pixel Detector, ATLAS Note, in preparation.

[10] The ATLAS Pixel Group, Pixel Offline Analysis for Endcap A Cosmic Data, ATLAS Note, ATLINDET-PUB-2008-001, 2008. 